\title{
The Prevalence of Malnutrition and Effectiveness of STRONGkids Tool in the Identification of Malnutrition Risks among Pediatric Surgical Patients
}

\author{
Çiğdem Ulukaya Durakbaşa, Selma Fettahoğlu, Ahu Bayar, Murat Mutus, Hamit Okur
}

Department of Pediatric Surgery, İstanbul Medeniyet University Faculty of Medicine, İstanbul, Turkey

\begin{abstract}
Background: High prevalence of malnutrition along with the risk for the development of malnutrition in hospitalised children has been reported. However, this problem remains largely unrecognised by healthcare workers.

Aims: To determine the prevalence of malnutrition and effectiveness of STRONGkids nutritional risk screening (NRS) tool in the identification of malnutrition risk among pediatric surgical patients.

Study Design: Cross-sectional study.

Methods: A total of 494 pediatric surgical patients (median age 59 months, $75.8 \%$ males) were included in this prospective study conducted over 3 months. SD-scores $<-2$ for Body Mass Index (BMI) for age or weight-for-height (WFH) and height-for-age (HFA) were considered to indicate acute and chronic malnutrition, respectively. The STRONGkids NRS tool was used to determine risk for malnutrition.

Results: Malnutrition was detected in $13.4 \%$ in this group of pediatric surgical patients. Acute malnutrition was identified in $10.1 \%$ of patients and more commonly in patients aged $\leq 60$ months than aged $>60$ months ( 13.4 vs. $6.6 \%, \mathrm{p}=0.012)$. Chronic malnutrition was identified in $23(4.6 \%)$ of patients with no significant difference between age groups. There were $7(1.4 \%)$ children with coexistent acute and chronic malnutrition. The STRONGkids tool revealed that $35.7 \%$ of patients were either in the moderate or high risk group for malnutri-
\end{abstract}

tion. Malnutrition, as revealed by anthropometric measurements, was more likely in the presence of gastrointestinal $(26.9 \%, \mathrm{p}=0.004)$ and inguinoscrotal/penile surgery $(4.0 \%, \mathrm{p}=0.031)$, co-morbidities affecting nutritional status $(\mathrm{p}<0.001)$ and inpatient admissions $(\mathrm{p}=0.014)$. Among patients categorized as low risk for malnutrition, there were more outpatients than inpatients $(89.3$ vs. $10.7 \%, \mathrm{p}<0.001)$ and more elective surgery cases than emergency surgery cases (93.4 vs. $6.6 \%$, $\mathrm{p}<0.001)$.

Conclusion: Providing data on the prevalence of malnutrition and risk of malnutrition in a prospectively recruited group of hospitalised pediatric surgical patients, the data acquired in the present study emphasise the need to raise clinician's awareness about the importance of nutritional status assessment among hospitalised pediatric patients and the benefits of identifying patients at the risk of nutritional depletion before malnutrition occurs. Our findings support the use of the STRONGkids tool among pediatric surgical patients to identify patients at risk for malnutrition and to increase the physician's awareness of nutritional assessment among hospitalised patients upon admission.

Key Words: Hospitalisation, malnutrition, nutritional status, outpatients, pediatric surgery, risk assessment, STRONGkids
Nutritional status in children has been considered an indicator of health and well-being at the individual and population levels (1). Both the malnutrition prevalence and the risk for development of malnutrition have been consistently reported as high in hospitalised children $(2,3)$. However, this problem remains largely unrecognised by healthcare workers. Due to the likelihood of the prevention of nutrition-associated complications, such as the slowing of growth and increased susceptibility to various infections, as well as prolonged hospi- talisation, early detection of the risk for malnutrition among hospitalised children has been considered essential $(3,4)$.

The importance of identifying those children at increased nutritional risk has led to the development of a number of nutritional risk screening (NRS) tools. However, there is a paucity of data on their application in clinical practice and the degree of inter-tool agreement and a consensus regarding which screening tool to use has not yet been reached $(3,5)$. The most recent instrument, STRONGkids, has been developed accord-

This study was presented at the 14 $4^{\text {th }}$ Congress of the European Pediatric Surgeons'Association (EUPSA), 5-8 June 2013, Leipzig, Germany.

Address for Correspondence: Dr. Çiğdem Ulukaya Durakbaşa, Department of Pediatric Surgery, İstanbul Medeniyet University Faculty of Medicine, İstanbul, Turkey Phone: +90 5322530569 E-mail: cigdemulukaya@yahoo.com

Received: 03.05.2014 Accepted: 27.09.2014 • DOI: 10.5152/balkanmedj.2014.14374

Available at www.balkanmedicaljournal.org

Cite this article as:

Durakbaşa ÇU, Fettahoğlu S, Bayar A, Mutus M, Okur H. The prevalence of malnutrition and effectiveness of STRONGkids tool in the identification of malnutrition risks among pediatric surgical patients. Balkan Med J 2014;31:313-21. 
ing to the newest European Society for Parenteral and Enteral Nutrition (ESPEN) guidelines in an effort to introduce an easy to apply NRS tool to overcome some of the issues reported for previous tools, such as the fact that they were too complicated and time-consuming to use in daily clinical practice $(2,6,7)$. The STRONGkids tool is a comprehensive summary of commonly asked questions concerning nutritional issues, combined with a clinical view of the child's status; as this is performed upon admission to the hospital, it helps to raise the clinician's awareness of nutritional risks (7).

The present study was designed to determine the prevalence of malnutrition and the effectiveness of STRONGkids NRS tool in the identification of malnutrition risk among pediatric surgical patients.

\section{MATERIALS AND METHODS}

\section{Study population}

This prospective study was conducted in a single pediatric surgery unit of a tertiary referral hospital over three consecutive months between April and July 2012. Patients who were younger than 30 days of age, admitted to the clinics other than "pediatric surgery" ward or had had another operation in the preceding 30 days were excluded. All of the remaining patients who were operated on during the given time interval were included. By definition, outpatients were those children who were admitted to the hospital for surgery but stayed for less than 24 hours.

Written informed consent was obtained from each subject or relative following a detailed explanation of the objectives and protocol of the study which was conducted in accordance with the ethical principles stated in the "Declaration of Helsinki" and approved by the institutional ethics committee.

\section{Definition of malnutrition}

Malnutrition was evaluated based on anthropometric measurements that were performed by the same ward nursing staff. WHO Anthro and AnthroPlus Programs were used for the evaluation of results $(8,9)$. For acute malnutrition (AM), Weight-for-Height (WFH) Z score or Body Mass Index (BMI) for age $Z$, scores of $\geq-3$ to $<-2$ were considered moderate malnutrition and scores of $<-3$ as severe malnutrition, while scores of $\geq-2$ denoted a lack of AM (10). For chronic malnutrition (CM), Height-for-Age (HFA) $\mathrm{Z}$ scores of $\geq-3$ to $<-2$ were deemed moderate malnutrition and scores of $<-3$ as severe malnutrition, while scores of $\geq-2$ denoted a lack of $\mathrm{CM}$ in accordance with WHO classification (10). Mid-upper arm circumference (MUAC) Z scores were calculated for patients aged $\leq 60$ months only, in compliance with WHO standards.

\section{STRONGkids nutritional risk screening tool}

Risk for malnutrition was evaluated via the STRONGkids questionnaire (7), which was completed by physicians via the face to face method. STRONGkids is a malnutrition "risk assessment tool" that consists of 4 items, including subjective clinical assessment (1 point), high risk disease (2 points), nutritional intake and losses (1 point) and weight loss or poor weight gain (1 point).

Patients with STRONGkids score 0 were classified as "low risk", whereas those with a score of 1-3 were classified as "medium risk" and those with a score of 4-5 were classified as "high risk".

\section{Statistical analysis}

Statistical analysis was performed using computer software (IBM SPSS Statistics version 20.0, SPSS Inc. Chicago, IL, USA). Categorical variables were compared with the Chisquare, Fisher's exact or Mantel Haenszel tests. Continuous variables were compared with the Kruskal Wallis and Mann Whitney U tests. Data were expressed as "mean (standard deviation; SD)", minimum-maximum and percent (\%) where appropriate. A $\mathrm{p}<0.05$ was considered statistically significant.

\section{RESULTS}

\section{Patient demographics and basic clinical features}

A total of 494 pediatric surgical patients were included in this study. The study population was composed primarily of male patients $(75.8 \%)$ with a mean age of 70.1 (SD 56.1 , range 1-220) months. The percentage of patients aged $\leq 60$ months was $51.1 \%$ while $48.9 \%$ were aged $>60$ months. Elective surgery was performed in $76.0 \%$ and outpatient admission was evident in $67.0 \%$ of patients. Among the inpatients, $63.8 \%$ were admitted for $\leq 3$ days and the remaining $36.2 \%$ were admitted for over 3 days. The admission rate for those remaining in hospital for over 3 days was $11.7 \%$ when all patients were considered. Inguinoscrotal and penile diseases $(50.3 \%)$ were the most common primary operative indication. Co-morbidities affecting nutritional status like chronic renal failure, swallowing dysfunction and the presence of malignancy were present in $16.4 \%$ (Table 1 ). In contrast, co-morbidities not affecting nutritional status like unilateral renal agenesis, minor cardiac malformations or Familial Mediterranean Fever under medical therapy were present in $4 \%$ of cases.

\section{Anthropometrics and $\mathbf{Z}$ scores}

Mean BMI was 16.8 (SD 3.2, ranged 9.5-33.6) $\mathrm{kg} / \mathrm{m}^{2}$ and MUAC was 18.5 (SD 4.3, range 9.0-36.0) $\mathrm{cm}$. in the overall 
TABLE 1. Patient demographics and basic clinical features $(n=494)$

\begin{tabular}{|c|c|c|c|}
\hline Age & $\mathrm{n}(\%)$ & Mean (SD) & Median (min-max) \\
\hline Overall (months) & $494(100.0)$ & $70.1(56.1)$ & $59.0(1.0-220.0)$ \\
\hline$\leq 60$ months & $253(51.1)$ & $25.1(17.4)$ & $22.0(1-60)$ \\
\hline$>60$ months & $241(48.9)$ & $116.6(42.7)$ & $106.5(61.0-220.0)$ \\
\hline Gender & & $\mathrm{n}(\%)$ & \\
\hline Male & & $374(75.8)$ & \\
\hline Female & & $120(24.2)$ & \\
\hline \multicolumn{4}{|l|}{ Type of surgery } \\
\hline Emergency & & $119(24)$ & \\
\hline Elective & & $375(76)$ & \\
\hline \multicolumn{4}{|l|}{ Admitted clinic setting } \\
\hline Outpatient & & $334(67.0)$ & \\
\hline Inpatient & & $160(33.0)$ & \\
\hline For 1-3 days & & $102(63.8)$ & \\
\hline For $>3$ days & & $58(36.2)$ & \\
\hline \multicolumn{4}{|c|}{ Primary operative indication } \\
\hline Inguinoscrotal and & le surgery & $248(50.3)$ & \\
\hline Acute abdominal s & & $94(19.0)$ & \\
\hline Urological surgery & & $34(6.9)$ & \\
\hline Gastrointestinal su & & $26(5.3)$ & \\
\hline Foreign body inges & aspiration & $23(4.6)$ & \\
\hline Other & & $69(13.9)$ & \\
\hline Other minor & & $63(12.7)$ & \\
\hline Oncologic surgery & & $3(0.6)$ & \\
\hline Thoracic surgery & & $3(0.6)$ & \\
\hline \multicolumn{4}{|l|}{ Co-morbidities } \\
\hline \multicolumn{4}{|c|}{ Affecting nutritional status } \\
\hline Positive & & $81(16.4)$ & \\
\hline Negative & & $413(83.6)$ & \\
\hline \multicolumn{4}{|c|}{ Not affecting nutritional status } \\
\hline Positive & & $20(4.0)$ & \\
\hline Negative & & $474(96.0)$ & \\
\hline \multicolumn{4}{|l|}{ History of prematurity } \\
\hline Yes & & $15(3.0)$ & \\
\hline No & & $479(97.0)$ & \\
\hline
\end{tabular}

population. Mean z scores for weight for age, height for age, BMI for age and weight for height were 0.3 (SD 1.6, range $-5.1-9.4), 0.5$ (SD 1.9, range -10.9-11.6), -0.1 (SD 1.6, range $-6.7-5.9$ ), and -0.2 (SD 1.5, ranged -6.3-4.0), respectively. The mean MUAC Z score was 0.7 (SD 1.3, range -4.9-4.6) (Table 2). Anthropometrics and $Z$ scores as well as respective distribution with regard to age groups ( $\leq 60$ months and $>60$ months) are summarised in Table 2.

\section{Malnutrition prevalence}

Overall, malnutrition was present in $66(13.4 \%)$ patients (Table 3). The percentage of children with malnutrition was higher in patients aged $\leq 60$ months than in those aged $>60$ months $(16.6 \%$ vs. $10 \%, p=0.015)$. Among the patients with malnutrition, 7 (1.4\%) had coexistent AM and CM.

AM was significantly more common in patients aged $\leq 60$ months compared with patients aged $>60$ months (13.4 vs. $6.6 \%, \mathrm{p}=0.012$ ), but there was no significant difference between age groups in terms of prevalence of CM (Table 3).

\section{STRONGkids risk groups}

The STRONGkids NRS tool revealed that most patients $(64.2 \%)$ were in the low risk category, while $34.5 \%$ were medium risk and only $1.2 \%$ were considered high risk for malnutrition (Table 3).

\section{The relation between malnutrition type and STRONGkids risk groups}

The rate of AM was determined to increase from $8.2 \%$ in patients at low risk to $33.3 \%$ in patients at high risk for malnutrition ( $\mathrm{p}=0.026$ ). $\mathrm{CM}$ was noted in $3.5 \%$ of patients at low risk, while it was reported in $16.7 \%$ of those at high risk $(\mathrm{p}=0.057)$. There was a statistically significant relationship $(\mathrm{p}=0.026)$ and a borderline significant trend $(\mathrm{p}=0.057)$ between overall future risk for malnutrition and the presence of $\mathrm{AM}$ and $\mathrm{CM}$, respectively. There was no significant difference between risk groups in terms of the presence of malnutrition (Table 4).

\section{Moderate to severe acute/chronic malnutrition in relation to study parameters}

Moderate to severe AM and CM were determined to be more likely in the presence of gastrointestinal $(26.9 \%, \mathrm{p}=0.004)$ and inguinoscrotal $/$ penile surgery $(4.0 \%, \mathrm{p}=0.031)$, co-morbidities affecting nutritional status $(\mathrm{p}<0.001)$, and in inpatient than outpatient admission $(\mathrm{p}=0.014)$. CM was more common in patients with a history of prematurity (corrected gestational age $\leq 6$ months) $(\mathrm{p}=0.003)$ (Table 5).

Having MUAC Z scores of $<-2$ was also more likely in the presence of gastrointestinal $(\mathrm{p}=0.025)$ and inguinoscrotal/penile surgery $(\mathrm{p}=0.031)$, co-morbidities affecting nutritional status $(\mathrm{p}=0.018)$ and history of prematurity $(\mathrm{p}=0.014)$ (Table 5).

\section{STRONGkids risk groups with respect to study parameters}

Patients determined to be in the medium risk category for malnutrition according to the STRONGkids risk assessment tool were significantly older (100.0 months) than patients in the low (53.6 months) and high (52.7 months) risk categories $(p<0.001)$. Mean $(\mathrm{SD})$ HAZ scores were 
TABLE 2. Anthropometrics and $\mathrm{Z}$ scores with respect to age groups

\begin{tabular}{|c|c|c|c|c|c|c|c|c|c|}
\hline \multirow[b]{2}{*}{ Anthropometrics } & \multicolumn{3}{|c|}{ Age $\leq 60$ months $(\mathrm{n}=253)$} & \multicolumn{3}{|c|}{ Age $>60$ months $(\mathrm{n}=241)$} & \multicolumn{3}{|c|}{ Total $(n=494)$} \\
\hline & $\mathrm{n}$ & Mean (SD) & Median (min-max) & $\mathrm{n}$ & Mean (SD) & Median (min-max) & $\mathrm{n}$ & Mean (SD) & Median (min-max) \\
\hline Weight (kg) & 253 & $12.3(4.5)$ & $12.0(3.3-25.0)$ & 241 & $34.9(15.9)$ & $30.1(12.0-91.7)$ & 494 & $23.3(16.2)$ & $18.5(3.3-91.7)$ \\
\hline Height $(\mathrm{cm})$ & 253 & $86.4(16.3)$ & $88.0(47.0-118.0)$ & 241 & $136.8(19.2)$ & $134.0(99.0-176.0)$ & 494 & $111.0(30.9)$ & $110.0(47.0-176.0)$ \\
\hline Body mass index $\left(\mathrm{kg} / \mathrm{m}^{2}\right)$ & 253 & $15.9(2.0)$ & $15.8(9.5-23.1)$ & 241 & $17.7(3.9)$ & $16.6(10.2-33.6)$ & 494 & $16.8(3.2)$ & $16.1(9.5-33.6)$ \\
\hline $\begin{array}{l}\text { Mid-upper arm } \\
\text { circumference }(\mathrm{cm})\end{array}$ & 253 & $15.9(2.1)$ & $16.0(9.0-23.0)$ & 239 & $21.2(4.4)$ & $20.0(13.0-36.0)$ & 492 & $18.5(4.3)$ & $17.0(9.0-36.0)$ \\
\hline \multicolumn{10}{|l|}{$Z$ scores } \\
\hline Weight for age & 253 & $0.3(1.6)$ & $0.3(-5.1-9.4)$ & 147 & $0.3(1.6)$ & $0.3(-4.4-5.2)$ & 400 & $0.3(1.6)$ & $0.3(-5.1-9.4)$ \\
\hline Height for age & 253 & $0.8(2.1)$ & $0.6(-6.5-11.6)$ & 241 & $0.1(1.3)$ & $0.3(-4.2-3.2)$ & 494 & $0.5(1.9)$ & $0.5(-10.9-11.6)$ \\
\hline Body mass index for age & 253 & $-0.3(1.5)$ & $-0.2(-6.7-253)$ & 241 & $0.1(1.6)$ & $0.1(-5.3-241)$ & 494 & $-0.1(1.6)$ & $-0.1(-6.7-5.9)$ \\
\hline Weight for height & 253 & $-0.2(1.5)$ & $-0.1(-6.3-4.0)$ & - & - & - & 253 & $-0.2(1.5)$ & $-0.1(-6.3-4.0)$ \\
\hline $\begin{array}{l}\text { Mid-upper arm } \\
\text { circumference for age }\end{array}$ & 231 & $0.7(1.3)$ & $0.6(-4.9-4.6)$ & - & - & - & 231 & $0.7(1.3)$ & $0.6(-4.9-4.6)$ \\
\hline
\end{tabular}

TABLE 3. Malnutrition prevalence with respect to age groups

\begin{tabular}{lcccc}
\hline & $\begin{array}{c}\text { Age } \leq 60 \text { months } \\
(\mathrm{n}=253)\end{array}$ & $\begin{array}{c}\text { Age }>60 \text { months } \\
(\mathrm{n}=241)\end{array}$ & $\begin{array}{c}\text { Total } \\
(\mathrm{n}=494)\end{array}$ & \\
\hline Malnutrition type & \multicolumn{1}{c}{$\mathrm{n}(\%)$} & $428(86.6)$ & 0.015 \\
Negative & $211(83.4)$ & $217(90)$ & $50(10.1)$ & 0.012 \\
Acute & $34(13.4)$ & $16(6.6)$ & $23(4.7)$ & 0.739 \\
Chronic & $11(4.3)$ & $12(5.0)$ & $59(11.9)$ & 0.015 \\
Only acute or chronic & $39(15.4)$ & $20(8.3)$ & $7(1.4)$ & 0.656 \\
Acute and chronic & $3(1.2)$ & $4(1.7)$ & & \\
\hline
\end{tabular}

TABLE 4. The relation between malnutrition type and STRONGkids risk groups

\begin{tabular}{lccccccc}
\hline Strongkids risk group & Low risk & Medium risk & High risk & Total & Comparison & Test & $\mathrm{p}$ value \\
\hline Acute malnutrition & & & $\underline{\mathrm{n}(\%)}$ & & All group & Mantel Haenszel & 0.026 \\
$\quad$ Negative & $292(91.8)$ & $148(87.1)$ & $4(66.7)$ & $444(89.9)$ & Low vs. medium & Chi square & $0.092^{*}$ \\
$\quad$ Positive & $26(8.2)$ & $22(12.9)$ & $2(33.3)$ & $50(10.1)$ & Low vs. high & Fisher's exact & $0.087^{*}$ \\
$\quad$ Total & $318(100.0)$ & $170(100.0)$ & $6(100.0)$ & $494(100.0)$ & Medium vs. high & Chi square & $0.190^{*}$ \\
Chronic malnutrition & & & $\underline{\mathrm{n}(\%)}$ & & All group & Mantel Haenszel & 0.057 \\
Negative & $307(96.5)$ & $159(93.5)$ & $5(83.3)$ & $471(95.3)$ & Low vs. medium & Chi square & $0.127^{*}$ \\
Positive & $11(3.5)$ & $11(6.5)$ & $1(16.7)$ & $23(4.7)$ & Low vs. high & Fisher's exact & $0.204^{*}$ \\
Total & $318(100.0)$ & $170(100.0)$ & $6(100.0)$ & $494(100.0)$ & Medium vs. high & Chi square & $0.350^{*}$ \\
\hline
\end{tabular}

*Significance level (type 1 error) of sub-group comparisons was set to $<0.167$ by using Bonferroni adjustment

significantly lower in the high risk (-1.62 (2.15)) and moderate risk $(0.07(1.70))$ groups when compared to the low risk group $(0.73(1.80))(\mathrm{p}<0.001)$ (Table 6). With regard to patients categorised as low risk for malnutrition, outpatients were significantly more common than inpatients ( 89.3 vs. $10.7 \%, p<0.001)$ and elective surgery was significantly more common than emergency surgery (93.4 vs. $6.6 \%, \mathrm{p}<0.001$ ) in this group (Table 6).

\section{DISCUSSION}

Malnutrition prevalence in pediatric surgical patients has not been sufficiently studied. The present study is unique, as it was performed in a group of Turkish pediatric surgical patients. It revealed he presence of malnutrition in $13.4 \%$ of the overall study population. AM was more common in patients aged $\leq 60$ months. Moderate to severe AM and CM were more likely in 
TABLE 5. Malnutrition/anthropometrics with respect to study parameters

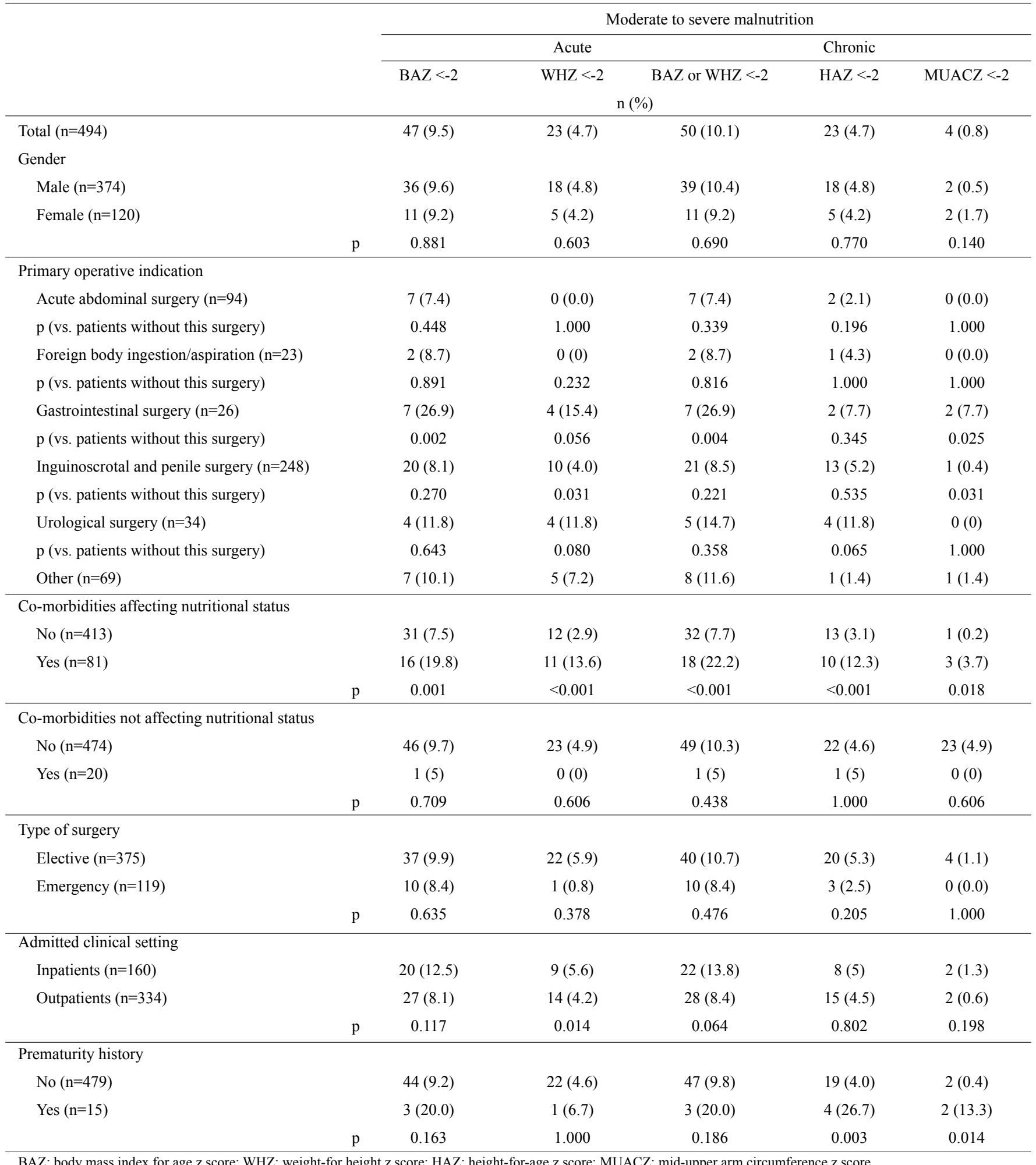


TABLE 6. STRONGkids risk groups with respect to study parameters

\begin{tabular}{|c|c|c|c|c|c|}
\hline \multicolumn{6}{|c|}{ STRONGkids risk group } \\
\hline & Low risk $(\mathrm{n}=318)$ & Medium risk $(\mathrm{n}=170)$ & High risk $(\mathrm{n}=6)$ & Total $(\mathrm{n}=494)$ & \multirow[b]{2}{*}{$\mathrm{p}$ value } \\
\hline & \multicolumn{4}{|c|}{ Mean (SD) } & \\
\hline Age (month) & $53.6(44.3)$ & $100.6(62.5)$ & $52.7(58.4)$ & $69.7(56.0)$ & $<0.001$ \\
\hline WHZ & $-0.08(1.38)$ & $-0.42(1.75)$ & $-1.38(2.52)$ & $-0.16(1.48)$ & 0.381 \\
\hline $\mathrm{BAZ}$ & $0.02(1.49)$ & $-0.16(1.66)$ & $-1.62(1.79)$ & $-0.06(1.56)$ & 0.085 \\
\hline HAZ & $0.73(1.80)$ & $0.07(1.70)$ & $-1.06(2.15)$ & $0.48(1.8)$ & $<0.001$ \\
\hline MUACZ & $0.82(1.16)$ & $0.37(1.52)$ & $-0.92(2.15)$ & $0.71(1.27)$ & 0.083 \\
\hline Admitted clinical setting & & $\mathrm{n}$ & & & \\
\hline Inpatients - $\mathrm{n}(\%)^{*}$ & $34(10.7)$ & $122(71.8)$ & $4(66.7)$ & $160(32.4)$ & \multirow{2}{*}{$<0.001$} \\
\hline Outpatient - n $(\%)^{*}$ & $284(89.3)$ & $48(28.2)$ & $2(33.3)$ & $334(67.6)$ & \\
\hline Type of surgery & \multicolumn{4}{|c|}{$\mathrm{n}(\%)$} & \multirow{3}{*}{$<0.001$} \\
\hline Elective- $n(\%) *$ & $297(93.4)$ & $73(42.9)$ & $5(83.3)$ & $375(75.9)$ & \\
\hline Emergency- n (\%)* & $21(6.6)$ & $97(57.1)$ & $1(16.7)$ & $119(24.1)$ & \\
\hline History of prematurity & \multicolumn{4}{|c|}{$\mathrm{n}(\%)$} & \\
\hline No- $\mathrm{n}(\%)^{*}$ & $315(99.1)$ & $159(93.5)$ & $5(83.3)$ & $479(97.0)$ & \multirow{2}{*}{$<0.001$} \\
\hline Yes- $\mathrm{n}(\%)^{*}$ & $3(0.9)$ & $11(6.5)$ & $1(16.7)$ & $15(3.0)$ & \\
\hline
\end{tabular}

"percentage in STRONGkids risk groups

BMI: body mass index Z score; WHZ: weight-for-height Z score; HAZ: height-for-age Z score; MUACZ: mid-upper arm circumference Z score

the presence of gastrointestinal and inguinoscrotal/penile surgery, co-morbidities affecting nutritional status and inpatient admissions. Higher prevalence of AM in children aged $<5$ years has been indicated in some other past studies (11).

Data from the Turkey Demographic and Health Survey in 2008 (12) revealed the prevalence of AM (WFA <-2SD) in $1.5 \%$ and $\mathrm{CM}(\mathrm{HFA}<-2 \mathrm{SD})$ in $7.5 \%$ of children aged $\leq 5$ years $(\mathrm{n}=396)$ in the general population. In another previous study performed among Turkish school children, AM and CM rates were reported to be $5.7 \%$ and $1 \%$, respectively, in children aged 6-16 years $(\mathrm{n}=1576)$ (13). Comparatively, we identified $\mathrm{AM}$ in $10.1 \%$ of pediatric surgical patients $(13.4 \%$ in patients aged $\leq 5$ years and $6.6 \%$ in patients aged $>5$ years) and $\mathrm{CM}$ in $4.7 \%(4.3 \%$ in patients aged $\leq 5$ years and $5.0 \%$ in patients aged $>5$ years). Identification of the higher prevalence of malnutrition among pediatric surgical patients than in the Turkish general pediatric population correlates with the statement that children who are admitted to hospital are at a higher risk of malnutrition than healthy children from the same community $(2,4,11,14)$.

Malnutrition rates of 31.8 to $56.6 \%$ in hospitalised pediatric patients have been previously reported in our country; these rates were much higher than the prevalence of AM reported in hospitalised children in Germany, France, UK, and the USA, with results varying from $6 \%$ to $14 \%(10,15-17)$ Accordingly, the prevalence of malnutrition in pediatric surgical patients on admission in the present study seems to be in agreement with data from developed countries, but poorer than rates reported in the Turkish general pediatric population and apparently lower than rates reported in pediatric hospitalised patients in Turkey $(10,14-17)$.

Malnutrition in hospitalised children has been very prevalent, especially in children with underlying disease and clinical conditions (2). Because AM and CM were significantly more likely in the presence of co-morbidities affecting nutritional status, our findings support previous studies indicating a higher prevalence of malnutrition in children with an underlying disease (14). In our study population, in addition to having co-morbidities affecting nutritional status, both AM and CM were determined to be more likely in the case of gastrointestinal and inguinoscrotal/penile surgery and a concomitant history of prematurity. In accordance with that, being a good indicator of the body's somatic muscle mass size, MUAC Z scores were lower in exactly the same patient groups. Given the presence of nutritional problems such as swallowing dysfunction due to esophageal stricture and achalasia as well as intestinal motility disorders, the higher likelihood of malnutrition in patients undergoing gastrointestinal surgery seems to be associated with the ongoing nutritional problems related to their primary diagnoses. In line with the consideration of preterm infants to be sensitive to changes in nutritional status with the frequent likelihood of growth failure during a hospital stay (2), malnutrition was noted to be more likely in the presence of prematurity history. On the other hand, the rela- 
tionship between malnutrition and inguinoscrotal/penile surgery in our patient population seems to be secondary to age rather than the underlying diagnosis. Indeed, AM as well as inguinoscrotal and penile pathologies were all more common in patients aged $\leq 60$ months.

Nutritional risk screening differs from global nutritional assessment in terms of identifying those individuals at risk of deterioration rather than those who are already malnourished. The NRS is a quick and simple process combining personal nutritional status with clinical disease information $(18,19)$. To date, four non-disease-specific nutrition screening tools designed for pediatrics have been developed for use. These are the Simple Pediatric Nutrition Risk Score (PNRS), Screening Tool for the Assessment of Malnutrition in Pediatrics (STAMP), Pediatric Yorkhill Malnutrition Score (PYMS) and STRONGkids (7, 20-22). The STRONGkids tool has been considered to be quicker to apply than PYMS and STAMP owing to the exclusion of weight and height measurements. It considers the impact of underlying diseases unlike PYMS and requires physician assessment, unlike both PYMS and STAMP which were designed for completion by nursing staff (11). The inter-tool agreement was found to be good between STAMP, STRONGkids and PNRS in one study conducted using 46 children with inflammatory bowel disease (23). None of these tools has been previously used in a population composed purely of pediatric surgical patients.

Although STRONGkids was developed to estimate the future risk of malnutrition in hospitalised patients, recent studies have used the tool in the outpatient setting as well (24). The initial study was conducted nationwide in Dutch pediatric wards in 2010 (7). It revealed that in a population with a malnutrition prevalence of $19 \%$, STRONGkids predicted that $54 \%$ of the children were at moderate risk and $8 \%$ were at high risk of developing malnutrition. Similarly, in our pediatric surgical patients, STRONGkids predicted that $34.5 \%$ were at moderate risk and $1.2 \%$ were at high risk of developing malnutrition. The increased risk for malnutrition determined via the STRONGkids risk assessment tool was significantly associated with a higher prevalence of both AM and CM. On the other hand, no statistical difference was noted in malnutrition prevalence with respect to individual risk groups.

Our findings support data from past studies reporting a correlation between STRONGkids risk categories and HFA Z scores $(3,7,11)$. However in contrast to past studies indicating a significant correlation of STRONGkids risk scores to WFH, HFA and BMI, no correlation was noted between anthropometric measures other than HZA and risk categories in our study population $(6,25,26)$.

Indeed, the lack of a relation between STRONGkids scores and anthropometric measurements apart from HZA in our study population also seems to be consistent with the fact that the STRONGkids NRS tool provides data on the future risk of malnutrition rather than the current nutritional status (7).

It was also reported that shorter stays at hospital were associated with the lack of a relationship between STRONGkids risk score and weight loss during hospital admission in pediatric ward patients (7). While the STRONGkids NRS tool revealed a high risk for malnutrition in only $1.2 \%$ of pediatric surgical patients in the present study, it should be noted that the majority of our inpatients were hospitalised for 1-3 days and the length of hospital stay was $>3$ days only in $11.7 \%$. Furthermore, inpatient admissions were associated with the increased likelihood of moderate to severe AM and CM compared with outpatient admissions. In accordance with that, STRONGkids assessment classified a significantly higher number of outpatients under the low risk category for future malnutrition than inpatients.

Malnutrition determined within the first $72 \mathrm{~h}$ of admission is mostlikelyattributabletoconditionspriortohospitalisation(27). The majority of our patients were hospitalised for less than 3 days. Therefore, hospital-acquired malnutrition does not seem to be an essential cause of the malnutrition observed in pediatric surgical patients. Although hospital-acquired malnutrition is important and should not be neglected in hospitalised pediatric patients, pediatric surgery wards seem to refer a specific group of patients in relation to the shorter length of hospital stay and higher likelihood of outpatient follow up.

A past study conducted with hospitalised pediatric patients emphasised that undernutrition on admission in children remains unrecognised by our healthcare workers, malnourished children are still not recognised sufficiently by pediatricians and that specific nutritional support is not used systematically (28). Especially, pediatric patients with mild malnutrition on admission were considered to be at the highest risk of adverse effect of hospitalisation and thus, this population of patients was recommended to be given special attention (28). Therefore, future efforts in pediatric nutrition should include identifying those patients who require nutritional support, ensuring the provision of appropriate nutritional management and educating hospital staff about the identification and management of malnutrition (16).

The use of any screening tool to identify children at risk of malnutrition can only be considered effective and reasonable if it results in early intervention and improved clinical outcomes. Much larger scale and longitudinal studies in hospitalised pediatric patients seem to be necessary to investigate whether or not malnutrition will develop in patients categorised to be at high risk for malnutrition via STRONGkids.

The principal strength of the present study is that it provides new information on the prevalence of malnutrition and risk 
of malnutrition in a prospectively recruited group of hospitalised Turkish pediatric surgical patients. The major limitation of this study is the representation of the cohort since it is a single-centre survey. However, it should be noted that by means of a single centre design, anthropometric measurements could be performed by the same nurse eliminating the influence of results by inter-observer variability. The second limitation seems to be the fact that the STRONGkids score does not include any objective assessment, whereas we also assessed the global nutritional statuses of the children by anthropometric examination. The third limitation of our study is the cross-sectional design with no data on the longitudinal analysis of nutritional changes over time which could limit the ability to identify whether those well-nourished patients who were classified as high risk by STRONGkids tool would have subsequently developed malnutrition.

In conclusion, the prevalence of malnutrition (13.4\%) and medium or high risk for malnutrition in $35.7 \%$ as determined by the NRS tool in the present study emphasises the need to raise clinician's awareness about the importance of nutritional status assessment among pediatric surgical patients and the benefits of identifying patients at risk of nutritional depletion before malnutrition occurs. Given the shorter length of hospital stay and the likelihood of hospital admission on an outpatient basis; factors other than hospital-acquired malnutrition, such as age $\leq 60$ months, type of surgery, co-morbidities effecting nutritional status and admitted clinical setting seem to be more effective in the prevalence and future risk of malnutrition among pediatric surgical patients. These findings support the use of an NRS tool among pediatric surgical patients to identify patients at risk for malnutrition and to increase physician's awareness about nutritional assessment among hospitalised patients on admission.

Acknowledgements: We thank to Çağla Ayhan, MD and Prof. Şule Oktay, $\mathrm{MD}, \mathrm{PhD}$ who provided editorial support and Mehmet Berktas, MD, MICR from KAPPA Consultancy Training Research Ltd, İstanbul who performed statistical analysis funded by Abbott Nutrition Turkey.

Ethics Committee Approval: Ethics committee approval was received for this study.

Informed Consent: Written informed consent was obtained from patients who participated in this study.

Peer-review: Externally peer-reviewed.

Author contributions: Concept - Ç.U.D., S.F., A.B., M.M., H.O.; Design - Ç.U.D., S.F., A.B.; Supervision - Ç.U.D., M.M., H.O.; Resource - Ç.U.D., S.F., A.B., M.M., H.O.; Materials - Ç.U. D., S F., A.B., M.M., H.O.; Data Collection\&/or Processing - Ç.U.D., S.F., A.B.; Analysis\&/or Interpretation - Ç.U.D., S.F., A.B., M.M., H.O.; Literature Search - Ç.U.D., S.F.; Writing Ç.U.D., S.F.; Critical Reviews - Ç.U.D., M.M., H.O.
Conflict of Interest: No conflict of interest was declared by the authors.

Financial Disclosure: The authors declared that this study has received no financial support.

\section{REFERENCES}

1. Widhalm K, Rashidian F, Emminger W_Huber WD, Bariss-Riedl M. Fritsch M, et al. Malnutrition in hospitalized children aged 3-18 years. Results by using a new score in comparison with previous described scores. J Ernährungsmedizin 2007;9:13-7.

2. Joosten KF, Hulst JM. Malnutrition in pediatric hospital patients: Current issues. Nutrition 2011;27:133-7. [CrossRef]

3. Huysentruyt K, Alliet P, Muyshont L, Rossignol R, Devreker T, Bontems P, Dejonckheere J, Vandenplas Y, De Schepper J. The STRONG nutritional screening tool in hospitalized children: A validation study. Nutrition 2013;29:1356-61. [CrossRef]

4. Joosten KFM, Hulst JM. Prevalence of malnutrition in pediatric hospital patients. Curr Opin Pediatr 2008;2:590-6. [CrossRef]

5. Wiskin AE, Owens DR, Cornelius VR, Wooton SA, Beattie RM. Paediatric nutrition risk scores in clinical practice: children with inflammatory bowel disease. J Hum Nutr Diet 2012;25:319-22. [CrossRef]

6. Kondrup J, Allison SP, Elia M, Vellas B, Plauth M. ESPEN guidelines for nutrition screening 2002. Clin Nutr 2003;22:415-21.[CrossRef]

7. Hulst JM, Zwart H, Hop WC, Joosten KF. Dutch national survey to test

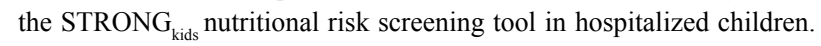
Clin Nutr 2010;29:106-11.[CrossRef]

8. WHO. Child growth standards. Available from: http://www.who.int/ childgrowth/software/en/

9. WHO. Growth reference 5-19 years. Available from: http://www.who. int/growthref/tools/en/

10. Joosten KF, Hulst JM. Prevalence of malnutrition in pediatric hospital patients. Curr Opin Pediatr 2008;20:590-6.[CrossRef]

11. Moeeni V, Walls T, Day AS. Assessment of nutritional status and nutritional risk in hospitalized Iranian children. Acta Paediatr 2012;101: e446-51.[CrossRef]

12. Turkey Demographic and Health Suvey 2008. Hacettepe University Institute of Population Studies, Ankara, Turkey, 2009. Available from: http://www.hips.hacettepe.edu.tr/eng/dokumanlar/TDHS-2008_Main_ Report.pdf

13. Gür E, Gunay C, Akkus S, Ercan G, Arvas A, Güzelöz S, Cifçili S. Is undernutrition a problem among Turkish School Children? Which factors have an influence on it? J Trop Pediatr 2006;52;421-6.[CrossRef]

14. Pawellek I, Dokoupil K, Koletzko B. Prevalence of malnutrition in paediatric hospital patients. Clin Nutr 2008;27:72-6.[CrossRef]

15. Özer N, Urganci N, Usta A, Kayaalp N. Determination of malnutrition in hospitalized children. T Klin J Pediatr 2001;10:133-8.

16. Doğan Y, Erkan T, Yalvaç S, Altay S, Cokuğraş FC, Aydin A, Kutlu T. Nutritional status of patients hospitalized in pediatric clinic. Turk J Gastroenterol 2005;16:212-6.

17. Hendricks KM, Duggan C, Gallagher L, Carlin AC, Richardson DS, Collier SB, Simpson W, Lo C. Malnutrition in hospitalized pediatric patients. Current prevalence. Arch Pediatr Adolesc Med 1995;149:1118-22. [CrossRef]

18. Mueller C, Compher C, Ellen DM. A.S.P.E.N. clinical guidelines: nutrition screening, assessment, and intervention in adults. J Parenter Enteral Nutr 2011;35:16-24. [CrossRef]

19. Aurangzeb B, Whitten KE, Harrison B, Mitchell M, Kepreotes H, Sidler $\mathrm{M}$, et al. Prevalence of malnutrition and risk of under-nutrition in hospitalized children. Clin Nutr 2012;31:35-40. [CrossRef] 
20. Sermet-Gaudelus I, Poisson-Salomon AS, Colomb V, Brusset MC, Mosser F, Berrier F, et al. Simple pediatric nutritional risk score to identify children at risk of malnutrition. Am J Clin Nutr 2000;72:64-70.

21. McCarthy HMH, Dixon M, Eaton-Evans MJ. Nutrition screening in children - the validation of a new tool. J Hum Nutr Diet 2008;21:395-6. [CrossRef]

22. GerasimidisK, Keane O, Macleod I, Flynn DM, Wright CM. A four-stage evaluation of the Paediatric Yorkhill Malnutrition Score in a tertiary paediatric hospital and a district general hospital. Br J Nutr 2010;104:751-6. [CrossRef]

23. Wiskin AE, Owens DR, Cornelius VR, Wootton SA, Beatie RM. Pediatric nutrition risk scores in clinical practice: children with inflammatory bowel disease. J Hum Nutr Diet 2012;25:319-22.[CrossRef]

24. Alliet PL, Verspeek L, Devolder H, Aerssens P, Achten W. Undernutrition in ambulatory children with cerebral palsy: anthropometrics vs STRONG $_{\text {kids }}$ Nutritional Screening Tool [abstract]. In: Szajewska H, Heyman MB, editors. Abstracts from the $47^{\text {th }}$ Annual Meeting of the
European Society for Paediatric Gastroenterology, Hepatology and $\mathrm{Nu}-$ trition; 2014 June 9-12; Jerusalem, Israel. J Pediatr Gastroenterol Nutr 2014;58(Suppl 1): S519.

25. Cao J, Peng L, Li R, Chen Y, Li X, Mo B, Li X. Nutritional risk screening and its clinical significance in hospitalized children. Clin Nutr 2014;33:432-6. [CrossRef]

26. Agostoni C, Axelson I, Colomb V, Goulte O, Koletzko B, Michaelsen KF, Puntis JW, Rigo J, Shamir R, Szajewska H, Turck D. The need for nutrition support teams in pediatric units: A commentary by the ESPGHAN Committee on Nutrition. J Pediatr Gastroenterol Nutr 2005;41:8-11.[CrossRef]

27. de Souza Menezes F, Leite HP, Koch Nogueira PC. Malnutrition as an independent predictor of clinical outcome in critically ill children. Nutrition 2012;28:267-70. [CrossRef]

28. Oztürk Y, Büyükgebiz B, Arslan N, Ellidokuz H. Effects of hospital stay on nutritional anthropometric data in Turkish children. J Trop Pediatr 2003;49:189-90.[CrossRef] 